\title{
A MINIMUM DEGREE CONDITION FOR FRACTIONAL ID-[a,b]-FACTOR-CRITICAL GRAPHS
}

\author{
SIZHONG ZHOU ${ }^{凶}$, ZHIREN SUN and HONGXIA LIU
}

(Received 21 September 2011)

\begin{abstract}
Let $G$ be a graph of order $n$, and let $a$ and $b$ be two integers with $1 \leq a \leq b$. Let $h: E(G) \rightarrow[0,1]$ be a function. If $a \leq \sum_{e \ni x} h(e) \leq b$ holds for any $x \in V(G)$, then we call $G\left[F_{h}\right]$ a fractional $[a, b]$-factor of $G$ with indicator function $h$, where $F_{h}=\{e \in E(G): h(e)>0\}$. A graph $G$ is fractional independent-setdeletable $[a, b]$-factor-critical (in short, fractional ID-[a,b]-factor-critical) if $G-I$ has a fractional $[a, b]$ factor for every independent set $I$ of $G$. In this paper, it is proved that if $n \geq((a+2 b)(a+b-2)+1) / b$ and $\delta(G) \geq((a+b) n) /(a+2 b)$, then $G$ is fractional ID- $[a, b]$-factor-critical. This result is best possible in some sense, and it is an extension of Chang, Liu and Zhu's previous result.
\end{abstract}

2010 Mathematics subject classification: primary 05C70.

Keywords and phrases: graph, minimum degree, independent set, fractional $[a, b]$-factor, fractional ID$[a, b]$-factor-critical graph.

\section{Introduction}

For motivation and background to this work, see [15]. Readers are referred to [1] for undefined terms and concepts. The graphs considered in this paper will be finite undirected graphs which have neither loops nor multiple edges. Let $G$ be a graph. We use $V(G)$ and $E(G)$ to denote its vertex set and edge set, respectively. For each $x \in V(G)$, we use $d_{G}(x)$ to denote the degree of $x$ in $G$, and $N_{G}(x)$ to denote the neighborhood of $x$ in $G$. We write $N_{G}[x]$ for $N_{G}(x) \cup\{x\}$. For $S \subseteq V(G)$, we denote by $G[S]$ the subgraph of $G$ induced by $S$, and $G-S=G[V(G) \backslash S]$. If $G[S]$ has no edges, then we call $S$ independent. The minimum degree of $G$ is denoted by $\delta(G)$. If $G_{1}$ and $G_{2}$ are disjoint graphs, the join and union are denoted by $G_{1} \vee G_{2}$ and $G_{1} \cup G_{2}$, respectively.

Let $a$ and $b$ be two positive integers with $1 \leq a \leq b$. Then a spanning subgraph $F$ of $G$ is called an $[a, b]$-factor if $a \leq d_{F}(x) \leq b$ for each $x \in V(G)$. If $a=b=k$, then

This research was supported by the Natural Science Foundation of the Higher Education Institutions of Jiangsu Province (10KJB110003), Jiangsu University of Science and Technology (2010SL101J) and Shandong Province Higher Educational Science and Technology Program (J10LA14), and was sponsored by the Qing Lan Project of Jiangsu Province.

(c) 2012 Australian Mathematical Publishing Association Inc. 0004-9727/2012 \$16.00 
an $[a, b]$-factor is called a $k$-factor. If $k=1$, then we say that a 1 -factor is a perfect matching. A graph $G$ is factor-critical [7] if $G-v$ has a perfect matching for each $v \in V(G)$. In [9], the concept of the factor-critical graph was generalised to the IDfactor-critical graph. We say that $G$ is independent-set-deletable factor-critical (in short, ID-factor-critical) if for every independent set $I$ of $G$ which has the same parity with $|V(G)|, G-I$ has a perfect matching. It is clear that every ID-factor-critical graph with odd vertices is factor-critical.

Let $h: E(G) \rightarrow[0,1]$ be a function. If $a \leq \sum_{e \ni x} h(e) \leq b$ holds for any $x \in V(G)$, then we call $G\left[F_{h}\right]$ a fractional $[a, b]$-factor of $G$ with indicator function $h$, where $F_{h}=\{e \in E(G): h(e)>0\}$. If $a=b=k$, then a fractional $[a, b]$-factor is called a fractional $k$-factor. A fractional 1-factor is also called a fractional perfect matching. A graph $G$ is fractional ID- $k$-factor-critical [2] if $G-I$ has a fractional $k$-factor for every independent set $I$ of $G$. In this paper, the concept of the fractional ID- $k$ factor-critical graph was generalised to the fractional ID- $[a, b]$-factor-critical graph, that is, a graph $G$ is fractional independent-set-deletable $[a, b]$-factor-critical (in short, fractional ID- $[a, b]$-factor-critical) if $G-I$ has a fractional $[a, b]$-factor for every independent set $I$ of $G$.

Many authors have investigated $[a, b]$-factors [3, 8, 10, 12, 13] and fractional factors [5, 6, 11, 14]. Chang et al. [2] obtained a minimum degree condition for a graph to be a fractional ID- $k$-factor-critical graph.

TheOREM 1.1 [2]. Let $k$ be a positive integer and $G$ be a graph of order $n$ with $n \geq 6 k-8$. If $\delta(G) \geq 2 n / 3$, then $G$ is fractional ID- $k$-factor-critical.

In this paper, we study fractional ID- $[a, b]$-factor-critical graphs, and obtain a minimum degree condition for a graph to be a fractional ID- $[a, b]$-factor-critical graph. Our main result is the following theorem, which is an extension of Theorem 1.1.

THEOREM 1.2. Let $G$ be a graph of order $n$, and let $a$ and $b$ be two integers with $1 \leq a \leq b$. If $n \geq((a+2 b)(a+b-2)+1) / b$ and $\delta(G) \geq((a+b) n) /(a+2 b)$, then $G$ is fractional ID-[a,b]-factor-critical.

\section{The proof of Theorem 1.2}

In order to prove Theorem 1.2, we rely heavily on the following lemma.

Lemma 2.1 [4]. Let $G$ be a graph. Then $G$ has a fractional $[a, b]$-factor if and only if for every subset $S$ of $V(G)$,

$$
\delta_{G}(S, T)=b|S|+d_{G-S}(T)-a|T| \geq 0,
$$

where

$$
T=\left\{x: x \in V(G) \backslash S, d_{G-S}(x) \leq a\right\} \quad \text { and } \quad d_{G-S}(T)=\sum_{x \in T} d_{G-S}(x) .
$$


Proof of Theorem 1.2. By Theorem 1.1, the result obviously holds for $a+b=2$ (that is, $a=b=1)$. In the following, we assume that $a+b \geq 3$. Let $X$ be an independent set of $G$ and $H=G-X$. Clearly, $|V(H)|=n-|X|, n-|X| \geq \delta(G)$ and $\delta(H) \geq \delta(G)-|X|$.

In order to complete the proof of Theorem 1.2, we need only to prove that $H$ has a fractional $[a, b]$-factor. By contradiction, we suppose that $H$ has no fractional $[a, b]$ factor. Then, according to Lemma 2.1, there exists some subset $S \subseteq V(H)$ such that

$$
\delta_{H}(S, T)=b|S|+d_{H-S}(T)-a|T| \leq-1 .
$$

We choose such subsets $S$ and $T$ so that $|T|$ is as small as possible.

Claim 1. We shall show that $d_{H-S}(x) \leq a-1$ for any $x \in T$.

Proof. If $d_{H-S}(x) \geq a$ for some $x \in T$, then the subsets $S$ and $T \backslash\{x\}$ satisfy (2.1), which contradicts the choice of $S$ and $T$. The proof of Claim 1 is complete.

$$
\begin{aligned}
& \text { Since } n-|X| \geq \delta(G) \text { and } \delta(G) \geq((a+b) n) /(a+2 b), \\
& \qquad \begin{aligned}
\frac{b(a+b)}{a^{2}}(\delta(G)-|X|)+\frac{b|X|}{a}-\frac{b n}{a} & =\frac{b(a+b) \delta(G)}{a^{2}}-\frac{b^{2}|X|}{a^{2}}-\frac{b n}{a} \\
& =\frac{b(a+b) \delta(G)}{a^{2}}+\frac{b^{2}}{a^{2}}(n-|X|)-\frac{b^{2} n}{a^{2}}-\frac{b n}{a} \\
& =\frac{b(a+b) \delta(G)}{a^{2}}+\frac{b^{2}}{a^{2}}(n-|X|)-\frac{b(a+b) n}{a^{2}} \\
& \geq \frac{b(a+b) \delta(G)}{a^{2}}+\frac{b^{2}}{a^{2}} \delta(G)-\frac{b(a+b) n}{a^{2}} \\
& =\frac{b(a+2 b) \delta(G)}{a^{2}}-\frac{b(a+b) n}{a^{2}} \\
& \geq \frac{b(a+2 b)}{a^{2}} \cdot \frac{(a+b) n}{a+2 b}-\frac{b(a+b) n}{a^{2}}=0,
\end{aligned}
\end{aligned}
$$

which implies

$$
\delta(G)-|X| \geq \frac{a}{a+b}(n-|X|) .
$$

Combining this with $\delta(H) \geq \delta(G)-|X|$,

$$
\delta(H) \geq \delta(G)-|X| \geq \frac{a}{a+b}(n-|X|) .
$$

Claim 2. We shall show that $|T| \geq b+1$.

Proof. According to (2.2),

$$
\begin{gathered}
n \geq \frac{(a+2 b)(a+b-2)+1}{b}>\frac{(a+2 b)(a+b-2)}{b}, \\
n-|X| \geq \delta(G) \text { and } \delta(G) \geq \frac{(a+b) n}{a+2 b},
\end{gathered}
$$


and

$$
\begin{aligned}
\delta(H) & \geq \frac{a}{a+b} \cdot \frac{(a+b) n}{a+2 b}=\frac{a n}{a+2 b}>\frac{a}{a+2 b} \cdot \frac{(a+2 b)(a+b-2)}{b} \\
& =\frac{a(a+b-2)}{b} \geq \frac{a(b-1)}{b}=a-\frac{a}{b} \geq a-1 .
\end{aligned}
$$

In terms of the integrity of $\delta(H)$,

$$
\delta(H) \geq a .
$$

If $|T| \leq b$, then, by (2.1) and (2.3),

$$
\begin{aligned}
-1 & \geq \delta_{H}(S, T)=b|S|+d_{H-S}(T)-a|T| \\
& \geq|T||S|+d_{H-S}(T)-a|T| \\
& =\sum_{x \in T}\left(|S|+d_{H-S}(x)-a\right) \\
& \geq \sum_{x \in T}(\delta(H)-a) \geq 0,
\end{aligned}
$$

which is a contradiction. This completes the proof of Claim 2.

According to Claim 2, $T \neq \emptyset$. Define

$$
h_{1}=\min \left\{d_{H-S}(x): x \in T\right\} .
$$

Choose $x_{1} \in T$ such that $d_{H-S}\left(x_{1}\right)=h_{1}$. If $T \backslash N_{T}\left[x_{1}\right] \neq \emptyset$, let

$$
h_{2}=\min \left\{d_{H-S}(x): x \in T \backslash N_{T}\left[x_{1}\right]\right\} .
$$

Choose $x_{2} \in T \backslash N_{T}\left[x_{1}\right]$ such that $d_{H-S}\left(x_{2}\right)=h_{2}$. According to Claim $1,0 \leq h_{1} \leq h_{2} \leq$ $a-1$. Obviously, $d_{H}\left(x_{i}\right) \leq|S|+h_{i}$ for $i=1,2$.

Case 1. $T=N_{T}\left[x_{1}\right]$.

Using Claim 2 and $T=N_{T}\left[x_{1}\right]$,

$$
a-1 \geq h_{1}=d_{H-S}\left(x_{1}\right) \geq\left|N_{T}\left[x_{1}\right]\right|-1=|T|-1 \geq b \geq a .
$$

This is a contradiction.

Case 2. $T \backslash N_{T}\left[x_{1}\right] \neq \emptyset$.

Note that $\left|N_{T}\left[x_{1}\right]\right| \leq d_{H-S}\left(x_{1}\right)+1=h_{1}+1$ and $a-h_{2} \geq 1$. Let $|V(H)|=p$. Then we obtain $p-|S|-|T| \geq 0$. Thus,

$$
\begin{aligned}
\left(a-h_{2}\right)(p-|S|-|T|)-1 & \geq \delta_{H}(S, T)=b|S|+d_{H-S}(T)-a|T| \\
& \geq b|S|+h_{1}\left|N_{T}\left[x_{1}\right]\right|+h_{2}\left(|T|-\left|N_{T}\left[x_{1}\right]\right|\right)-a|T| \\
& =b|S|-\left(a-h_{2}\right)|T|-\left(h_{2}-h_{1}\right)\left|N_{T}\left[x_{1}\right]\right| \\
& \geq b|S|-\left(a-h_{2}\right)|T|-\left(h_{2}-h_{1}\right)\left(h_{1}+1\right),
\end{aligned}
$$


that is,

$$
\left(a+b-h_{2}\right)|S| \leq\left(a-h_{2}\right) p+\left(h_{2}-h_{1}\right)\left(h_{1}+1\right)-1 .
$$

From (2.2) and $n-|X|=p$, we have $\delta(H) \geq a p /(a+b)$. Combining this with $|S| \geq \delta(H)-h_{1}$,

$$
|S| \geq \frac{a p}{a+b}-h_{1}
$$

According to (2.4) and (2.5),

$$
\left(a+b-h_{2}\right)\left(\frac{a p}{a+b}-h_{1}\right) \leq\left(a-h_{2}\right) p+\left(h_{2}-h_{1}\right)\left(h_{1}+1\right)-1,
$$

which implies

$$
(b p-a-b) h_{2} \leq(a+b)^{2} h_{1}-(a+b)\left(h_{1}+1\right) h_{1}-(a+b) .
$$

In terms of

$$
p=n-|X| \geq \delta(G), \quad \delta(G) \geq \frac{(a+b) n}{a+2 b}, \quad n \geq \frac{(a+2 b)(a+b-2)+1}{b}
$$

and $a+b \geq 3$, we get

$$
\begin{aligned}
b p \geq b \delta(G) \geq \frac{b(a+b) n}{a+2 b} & \geq \frac{(a+b)(a+2 b)(a+b-2)+(a+b)}{a+2 b} \\
& >(a+b)(a+b-2) \geq a+b .
\end{aligned}
$$

Combining this with (2.6) and $h_{1} \leq h_{2}$,

$$
(b p-a-b) h_{1} \leq(b p-a-b) h_{2} \leq(a+b)^{2} h_{1}-(a+b)\left(h_{1}+1\right) h_{1}-(a+b),
$$

that is,

$$
(a+b) h_{1}^{2}+\left(b p-(a+b)^{2}\right) h_{1}+(a+b) \leq 0 .
$$

Let $f\left(h_{1}\right)=(a+b) h_{1}^{2}+\left(b p-(a+b)^{2}\right) h_{1}+(a+b)$. If $h_{1}=0$, then, by (2.7), we have $2 \leq a+b \leq 0$, which is a contradiction. In the following, we may assume that $h_{1} \geq 1$. Since $b p>(a+b)(a+b-2)$,

$$
f^{\prime}\left(h_{1}\right)=2(a+b) h_{1}+b p-(a+b)^{2}>2(a+b)+(a+b)(a+b-2)-(a+b)^{2}=0 .
$$

Thus, by (2.7),

$$
\begin{aligned}
0 & \geq f\left(h_{1}\right) \geq f(1)=(a+b)+\left(b p-(a+b)^{2}\right)+(a+b) \\
& >2(a+b)+(a+b)(a+b-2)-(a+b)^{2}=0,
\end{aligned}
$$

which is a contradiction.

In each of the above cases we obtained contradictions. Hence, $H$ has a fractional $[a, b]$-factor, that is, $G$ is fractional ID- $[a, b]$-factor-critical.

This completes the proof of Theorem 1.2. 


\section{Remark}

In this section, we show that the condition $\delta(G) \geq((a+b) n) /(a+2 b)$ in Theorem 1.2 is sharp. To see this, we construct a graph $G=(a t) K_{1} \vee(b t) K_{1} \vee$ $(b t+1) K_{1}$, where $t$ is a sufficiently large positive integer. Obviously, $|V(G)|=n=$ $(a+2 b) t+1$ and

$$
\frac{(a+b) n}{a+2 b}>\delta(G)=(a+b) t=(a+b) \cdot \frac{n-1}{a+2 b}=\frac{(a+b) n}{a+2 b}-\frac{a+b}{a+2 b}>\frac{(a+b) n}{a+2 b}-1 .
$$

In the following, let $X=(b t) K_{1}$. Clearly, $X$ is an independent set of $G$. Put $H=G-X=(a t) K_{1} \vee(b t+1) K_{1}, S=(a t) K_{1}$ and $T=(b t+1) K_{1}$. Then

$$
\begin{aligned}
\delta_{H}(S, T) & =b|S|+d_{H-S}(T)-a|T| \\
& =a b t-a(b t+1)=-a<0 .
\end{aligned}
$$

According to Lemma 2.1, $H$ has no fractional $[a, b]$-factor. Hence, $G$ is not fractional ID- $[a, b]$-factor-critical. In the sense above, the bound of $\delta(G)$ in Theorem 1.2 is sharp.

\section{References}

[1] J. A. Bondy and U. S. R. Murty, Graph Theory with Applications (The Macmillan Press, London, 1976).

[2] R. Chang, G. Liu and Y. Zhu, 'Degree conditions of fractional ID- $k$-factor-critical graphs', Bull. Malays. Math. Sci. Soc. (2) 33(3) (2010), 355-360.

[3] Y. Li and M. Cai, 'A degree condition for a graph to have [a,b]-factors', J. Graph Theory 27 (1998), 1-6.

[4] G. Liu and L. Zhang, 'Fractional $(g, f)$-factors of graphs', Acta Math. Sci. Ser. B 21(4) (2001), 541-545.

[5] G. Liu and L. Zhang, 'Toughness and the existence of fractional $k$-factors of graphs', Discrete Math. 308 (2008), 1741-1748.

[6] G. Liu and L. Zhang, 'Characterizations of maximum fractional $(g, f)$-factors of graphs', Discrete Appl. Math. 156 (2008), 2293-2299.

[7] L. Lovasz and M. D. Plummer, Matching Theory (Elsevier Science, North-Holland, 1986).

[8] Y. Nam, 'Binding numbers and connected factors', Graphs Combin. 26(6) (2010), 805-813.

[9] J. Yuan, 'Independent-set-deletable factor-critical power graphs', Acta Math. Sci. Ser. B 26(4) (2006), 577-584.

[10] S. Zhou, 'Independence number, connectivity and ( $a, b, k)$-critical graphs', Discrete Math. 309(12) (2009), 4144-4148.

[11] S. Zhou, 'A minimum degree condition of fractional $(k, m)$-deleted graphs', $C$. R. Math. 347(21-22) (2009), 1223-1226.

[12] S. Zhou, 'A sufficient condition for a graph to be an $(a, b, k)$-critical graph', Int. J. Comput. Math. 87(10) (2010), 2202-2211.

[13] S. Zhou, 'Binding numbers and $[a, b]$-factors excluding a given $k$-factor', C. R. Math. 349(19-20) (2011), 1021-1024.

[14] S. Zhou, 'A sufficient condition for graphs to be fractional $(k, m)$-deleted graphs', Appl. Math. Lett. 24(9) (2011), 1533-1538.

[15] S. Zhou, Q. Bian and L. Xu, 'Binding number and minimum degree for fractional $(k, m)$-deleted graphs', Bull. Aust. Math. Soc. 85(1) (2012), 60-67. 
SIZHONG ZHOU, School of Mathematics and Physics, Jiangsu University of Science and Technology, Mengxi Road 2, Zhenjiang, Jiangsu 212003, PR China

e-mail: zsz_cumt@163.com

ZHIREN SUN, School of Computer Science, Nanjing Normal University, Nanjing, Jiangsu 210046, PR China

HONGXIA LIU, School of Mathematics and Informational Science, Yantai University, Yantai, Shandong 264005, PR China 